\title{
Modification of the Electrical Properties of Porous Silicon by Adsorption of Ammonia Molecules
}

\author{
I. B. Olenych ${ }^{1, *}$, L. S. Monastyrskii ${ }^{1}$, O. I. Aksimentyeva ${ }^{1}$, L. I. Yarytska ${ }^{2}$ \\ ${ }^{1}$ Ivan Franko National University of Lviv, 50 Dragomanov Str., 79005 Lviv, Ukraine \\ ${ }^{2}$ Lviv State University of Live Safety, 35 Kleparivska Str., 79000 Lviv, Ukraine \\ *Corresponding Author: iolenych@gmail.com
}

Copyright (C) 2014 Horizon Research Publishing All rights reserved.

\begin{abstract}
It was studied electrophysical characteristics of porous silicon (PS) layers formed on single-crystalline silicon substrates with both $n$ - and $p$-types of conductivity under conditions of adsorption of ammonia molecules. An increase in the conductivity of PS- $n$-Si structures was observed with increasing ammonia concentration. The adsorption of ammonia was shown to give rise to 'diode-like' current-voltage characteristics of PS- $p$-Si structures. Spectral characteristics of photovoltage and absorption bands in the infrared spectrum for our structures subjected to ammonia adsorption were studied. We suggested a possible mechanism for the influence of ammonia adsorption on the properties of the PS-silicon structures.
\end{abstract}

Keywords Porous Silicon, Ammonia Adsorption, Conductivity, Photovoltage, Infrared Spectroscopy

\section{Introduction}

Porous silicon (PS) reveals a number of unique properties caused by its sponge-like geometrical structure. Owing to an increased bandgap, antireflective properties and a large surface area per unit volume, silicon nanostructures are promising for photoelectronics and sensor electronics $[1,2]$. A common feature of most gas adsorption sensors based on the PS is usage of adsorption-induced electrical effects that cause substantial changes in the free charge carrier concentrations of the PS and its electrophysical parameters such as electrical conductivity or capacitance [3,4].

On the other side, controlled adsorptions of chemically active or polar molecules purposefully modify the electrical parameters of silicon nanostructures. It was observed transformation of hole-type conductivity of mesoporous silicon into electronic one (see the case of adsorption of $\mathrm{NH}_{3}$ molecules that exhibit donor properties [5]), or electronic-to-hole conductivity inversion (the case of adsorption of $\mathrm{I}_{2}$ or $\mathrm{Br}_{2}$ molecules revealing acceptor properties [6-8]). As a result, it was observed the appearance of photosensitive electrical barriers at the border PS - silicon substrate [7,8]. Nonetheless, applications of adsorption-based techniques for controlling the conductivity type have still not received sufficient attention from researchers.

It should be noted that by now there remains open the problem of doping of nanocryatals. Semiconductor nanocrystals exert resistance to introducing of doping impurities in them: on the one hand there takes place process of self-cleaning of nanocrystals from impurities, on the other hand, these impurities strongly distort the crystal lattice what gives rise to degradation of electrophysical parameters of nanostructures $[9,10]$. The problems with nanodoping are caused by the fundamental differences in mechanisms which control introduction of impurities in bulk materials and nanoparticles. If in macroscopic solids the main role belongs to thermodynamic issues which impose restrictions on the limiting concentration, then in nanocrystals always is determined by kinetics, and first of all by surface kinetics.

The detailed investigation of the features of doping of semiconductors nanocrystals is of interest since it opens a way to producing new types of semiconductor nanostructors for various applications. The purpose of the work was to investigate the possibility of control the type conductivity nanocrystals PS by adsorption of ammonia molecules. Proof of modification of electronic structure of silicon nanocrystals can be both the changing nature of electrical structures PS $\mathrm{Si}$ under the influence of adsorption of molecules $\mathrm{NH}_{3}$, and the emergence of photosensitive electric barriers.

\section{Experiment}

PS nanostructures were prepared by means of photoelectrochemical etching [11] on single-crystal substrates electronic and hole type conductivity with resistivity of 4.5 and $10 \mathrm{Ohm} \cdot \mathrm{cm}$ respectively. We used the ethanol solution of hydrofluoric acid (the volume ratio of the components $\left.\mathrm{HF}: \mathrm{C}_{2} \mathrm{H}_{5} \mathrm{OH}=1: 1\right)$ as an electrolyte. In order to obtain homogeneous layers, aluminium or silver films were preliminarily deposited on a back surface of the substrates with the aid of a thermo-vacuum technique. These films 
served also as contacts for further measurements. The anodic current density was equal to $30 \mathrm{~mA} / \mathrm{cm}^{2}$ and the etching time was $5 \mathrm{~min}$. Under the technological conditions mentioned above, mesoporous silicon layers were formed, with the thicknesses of about $10 \mu \mathrm{m}$. The structures were divided into samples with areas of about $1 \mathrm{~cm}^{2}$. Thus, it has been prepared two series of experimental samples of PS $-n$-Si and PS $-p$-Si. The contact with the diameter of $\sim 2 \mathrm{~mm}$ was deposited on the surface of porous layer, using colloidal carbon.

Modification of the electrical parameters of silicon nanocrystals was carried by adsorption of the molecules $\mathrm{NH}_{3}$. Control of process adsorption doping was carried out in an atmosphere of ammonia measuring electrophysical parameters of structures PS-Si in the modes of direct and alternating currents during the passage of current through the structure in the direction perpendicular to the surface. The measurements showed that electrical parameters of some samples of the series although being different in the range of $10 \%$ however the character of influence on them the ammonia molecules adsorption was the same.

Photoelectrical phenomena were studied after irradiating the structures from the direction of porous layer perpendicular to its surface. The light of a He-Ne laser was used for that purpose, with the wavelength $\lambda=0.63 \mu \mathrm{m}$ and the radiation power $2 \mathrm{~mW}$. The spectral dependences of the photoresponse were measured with a standard optical equipment based upon a diffraction monochromator and a filament lamp $(2800 \mathrm{~K})$. The photovoltage spectra were normalized to the curve of black-body radiation with the temperature of $2800 \mathrm{~K}$ and then corrected for spectral sensitivity of our setup. The spectral dependence of photoresponse of an industrial silicon photodiode was also measured for a comparison.

Changing the coverage of molecular nanostructures PS due to adsorption of ammonia molecules was investigated by infrared (IR) spectroscopy. Measurement of IR spectra was carried out on a spectrometer AVATAR in the $400-4000$ $\mathrm{cm}^{-1}$ range. Identification of absorption bands was carried out based on comparison with the literature data [12-15].

\section{Result and Discussion}

The reference samples of PS- $n-\mathrm{Si}$ and PS- $p$-Si had symmetrical and nonlinear current-voltage characteristics, indicating the existence in this structure of several potential barriers (Figure 1 and Figure 2).

Our studies reveal that the adsorption of ammonia molecules significantly affects the electrical conductivity of the 'PS-silicon substrate'. The natures of conductivity changes occurring in the PS- $n$-Si and PS- $p$-Si structures turn out to be different. An increase in the conductivity of PS- $n$-Si nanostructures is observed with increasing ammonia concentration (see Figure 1).

The CVC changes in the PS- $p$-Si structures occurring under the influence of ammonia adsorption are found to have a more complex character (see Figure 2). Apart from a decrease in the conductivity PS- $p$-Si observed with an increasing $\mathrm{NH}_{3}$ concentration, one can see a 'rectification effect' inherent to $p$ - $n$-junctions. Notice that the direct branch of the CVC corresponds in this case to the negative potential at the porous layer. This indicates predominance of one of the potential barriers.

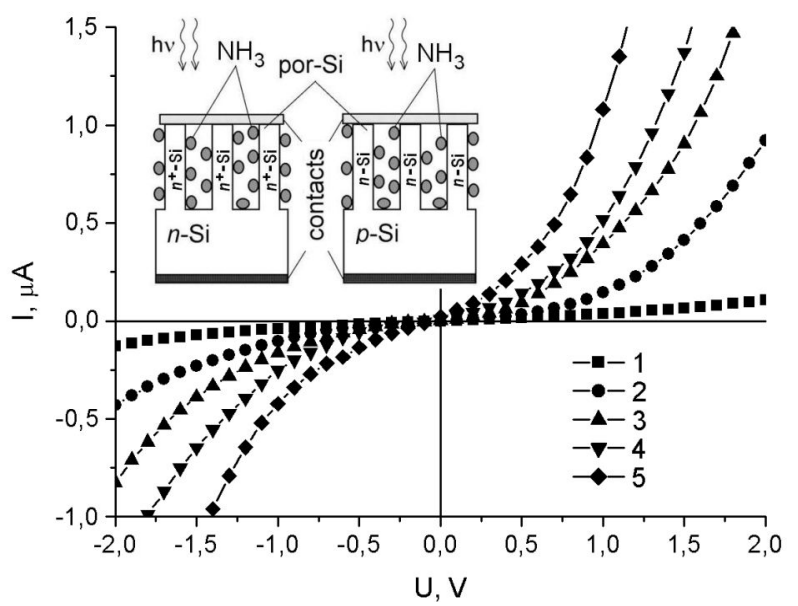

Figure 1. Dark CVC of the PS- $n$-Si structure at different concentrations of molecular ammonia: 0 - (1); 4,2\% - (2); 8,4\% - (3), 16,8\% - (4); 25,2\% (5). The inset shows scheme of samples with adsorbed $\mathrm{NH}_{3}$

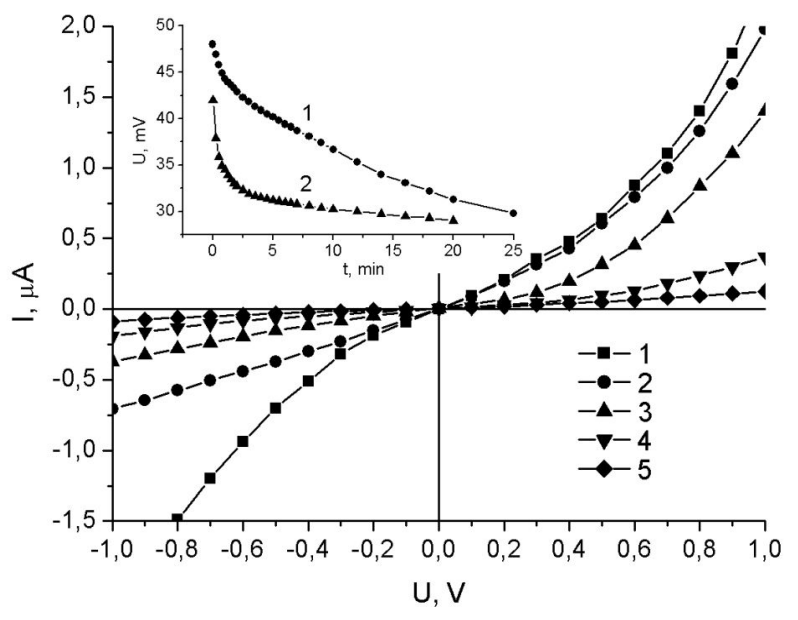

Figure 2. Dark CVC of the PS- $p$-Si structure at different concentrations of molecular ammonia: 0 - (1); 4,2\% - (2); 8,4\% - (3), 16,8\% - (4); 25,2\% (5). The inset shows relax of dark potential difference on the contacts PS- $n$-Si (1) and PS- $p$-Si (2) structures

In addition, the adsorption of ammonia molecules gives rise to nonzero value of the current at zero bias. The appearance of a dark potential difference under the influence of adsorption of ammonia was observed in [16] in studies of planar CVC. In our case, the dark potential differences between the contacts (positive sign in PS layer with respect to the silicon substrate) in open-circuit regime increase with increasing the concentration of ammonia, but relax with time (Figure 3). This is indicating electrets properties of experimental structures. Relaxation rate for PS- $p-\mathrm{Si}$ structure was higher compared with the structure of PS $-n-\mathrm{Si}$ (see inset in Figure 2). 


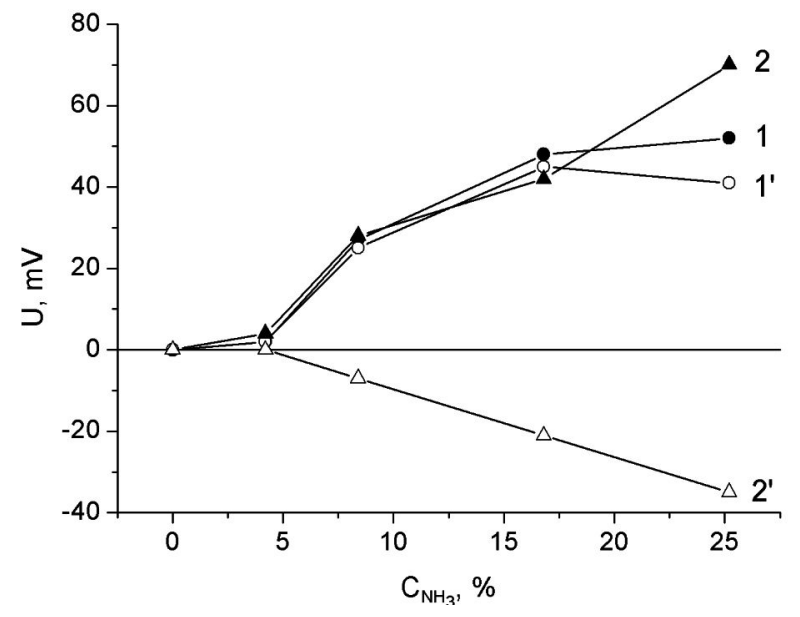

Figure 3. Dependence of the potential difference on the concentration of $\mathrm{NH}_{3}$ in the dark (curves 1 and 2) and under irradiation by the light of $\mathrm{He}-\mathrm{Ne}$ laser with the intensity of $60 \mathrm{~mW} / \mathrm{cm}^{2}$ (curves 1' and 2'). Curves 1, 1' refer to the PS- $n$-Si structures and curves 2,2 ' to the PS- $p$-Si ones

Under illumination the PS surface by He-Ne laser with an intensity of $60 \mathrm{~mW} / \mathrm{cm}^{2}$ it was observed a decrease in dark potential differences in the structure of PS- $n$-Si due to adsorption of molecules $\mathrm{NH}_{3}$ and recharge surface PS in structure PS- $p$-Si. Increase in the quantity of adsorbed ammonia molecules drove the value of photovoltage negative polarity at porous layer.

We have investigated the dependences of differential conductivity at the zero bias (measured at a signal testing frequency of $1 \mathrm{MHz}$ and amplitude $25 \mathrm{mV}$ ) on the ammonia concentration in the testing cell. As a matter of fact, increasing $\mathrm{NH}_{3}$ concentration leads to increase in the differential conductivity in the case of PS- $n$-Si structure and decreases the same parameter for the PS- $p$-Si structure (Figure 4). A slight increase in the differential conductivity due to photo-generated charge carriers is observed under illumination of the PS surface with the laser light.

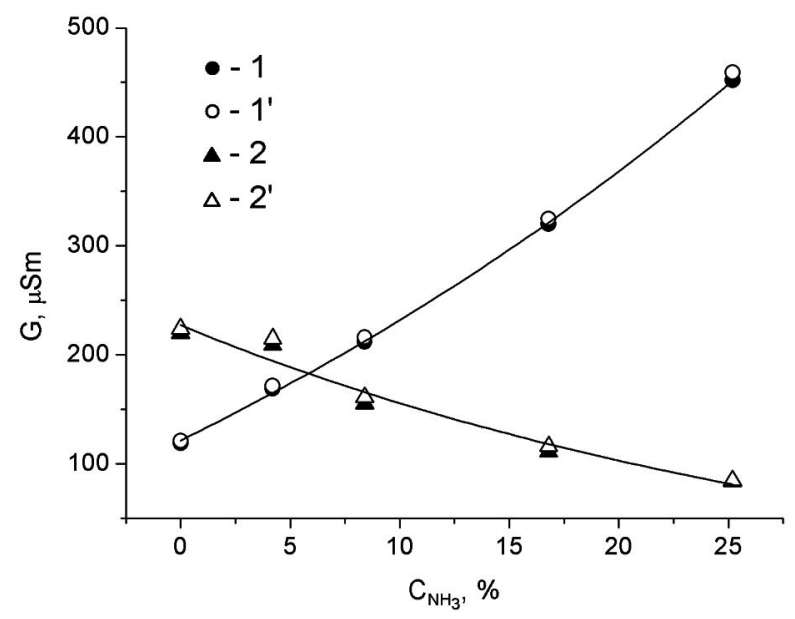

Figure 4. Dependences of differential conductivity at the zero bias obtained in the dark (curves 1 and 2) and under irradiation by the light of $\mathrm{He}-\mathrm{Ne}$ laser with the intensity of $60 \mathrm{~mW} / \mathrm{cm}^{2}$ (curves 1 ' and 2') on the concentration of $\mathrm{NH}_{3}$. Curves 1, 1' refer to the PS- $n$-Si structures and curves 2,2 ' to the PS- $p$-Si ones
The obtained results can be explained in the frame of the following qualitative model. The adsorption of molecular ammonia on the silicon surface induces formation of surface donor levels and the corresponding bending of energy bands. In its turn, the near-surface regions of $p$-Si and $n$-Si get respectively exhausted and enriched with the free charge carriers. Substantial changes in the carrier concentration can be expected under influence of ammonia molecular adsorption in the whole volume of PS nanocrystals, which are characterized by a large specific surface. Therefore the conductivities of the PS- $n$-Si and PS- $p$-Si structures should increase and decrease, respectively.

In the case of PS formed at $p$ - $\mathrm{Si}$, the adsorption of $\mathrm{NH}_{3}$ by the silicon nanocrystals reverses hole-type conductivity of the nanocrystals to the electronic type [5]. As a result, formation of electrical barriers (being analogous to the $p-n$-junctions in the silicon diodes) is observed at the PS- $p$-Si boundary. So, the structure of ammonia modified PS- $p$-Si can be considered as parallelly arranged $p$ - $n$-junctions (see inset in Figure 1). However, we can't exclude the existence of electrical barriers of other nature (for example, non-linearity of contact) photoconduction of porous layer and, consequently, more complex mechanisms of current passage in these structures.

For obtaining additional information about the nature of influence of the $\mathrm{NH}_{3}$ adsorption on photoelectrical properties of the PS- $p$-Si we have investigated spectral dependences of the photoresponse in that structure. The photovoltage spectra of modified samples at open circuit regime were similar to the photoresponse spectrum of industrial silicon photodiode and were characterized by the wide maximum in the $750-950 \mathrm{~nm}$ range (Figure 5).

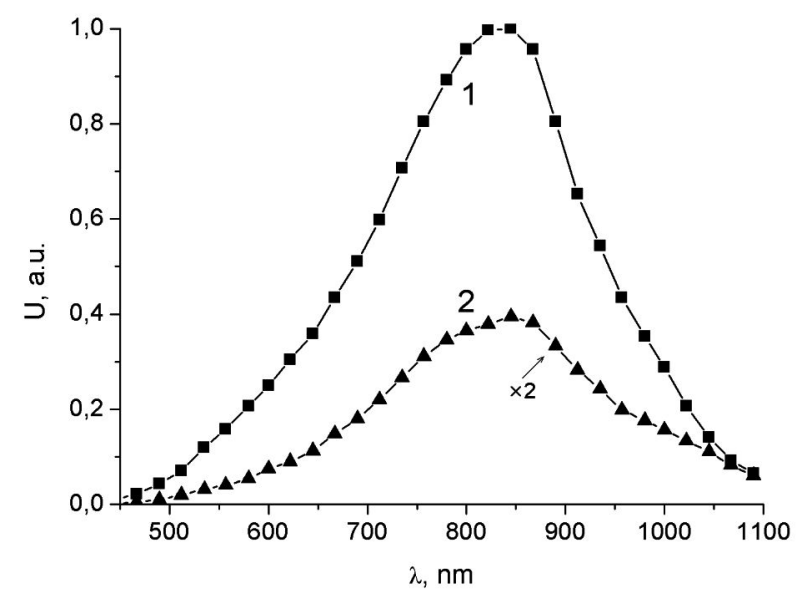

Figure 5. Photovoltage spectra for the industrial silicon photodiode (1) and PS- $p$-Si (2) measured at the $\mathrm{NH}_{3}$ concentration $25,2 \%$

A similarity of the spectral dependence of photoresponse of ammonia modified PS- $p$-Si structure with that of silicon photodiode allows us to suggest that the photovoltage is associated with light absorption in the silicon substrate and separation of photocarriers at the boundary of silicon-PS. 
Under illumination in the open-circuit regime, the photo-generated electron-hole pairs are separated by a potential barrier. During this process the electrons are accumulated in the PS nanocrystals, thus creating a potential at the porous layer, which is negative with respect to that of the silicon substrate.

Change of component on the PS surface due to adsorption of ammonia molecules was studied by analyzing the IR transmission spectra of experimental structures 'PS-silicon substrate'. The most of absorption bands are related to the molecular complexes containing hydrogen and oxygen (Figure 6). The most marked absorption bands were those at frequencies of 620 and $860 \mathrm{~cm}^{-1}$ corresponding to the $\mathrm{Si}-\mathrm{H}_{2}$ and $\mathrm{Si}-\mathrm{OH}$ deformation modes respectively, and at a frequency of $1100 \mathrm{~cm}^{-1}$ corresponding to valence oscillations of $\mathrm{Si}-\mathrm{O}-\mathrm{Si}$. In addition, the IR spectra of PS possessed the bands in the $2350-2400 \mathrm{~cm}^{-1}$ range, which are related to oscillations of $\mathrm{O}_{3}-\mathrm{Si}-\mathrm{H}$. The wide absorption band in the $3500-3960 \mathrm{~cm}^{-1}$ range is related to oscillations of $\mathrm{O}-\mathrm{H}$ bonds in water molecules which are adsorbed on the surface of PS. In the same spectral range there were observed the absorption bands that are related to the hydroxyl groups of $\mathrm{Si}-\mathrm{OH}$ and the molecular complexes of $\mathrm{Si}-\mathrm{H}_{2}, \mathrm{Si}-\mathrm{H}_{3}$.

Adsorption of ammonia molecules by porous silicon leads to a decrease in transmission of infrared radiation over the entire range of measurements. In addition it was observed the appearance of new absorption bands in the spectral regions $1450-1500 \mathrm{~cm}^{-1}$ and $1600-1800 \mathrm{~cm}^{-1}$ which are associated with the stretching oscillations of molecules $\mathrm{NH}_{3}$, adsorbed on the surface of silicon nanocrystals. Also it was observed some redistribution of the intensity of shoulder bands in the $1100 \mathrm{~cm}^{-1}$ range and shift of the peaks, which proves the increasing proportion of the surface oxide and the transformation component of the porous layer.

Changing the surface of the PS due to adsorption of molecules $\mathrm{NH}_{3}$ and can be cause of the electret properties of modified PS-Si structures, which were reported in the study of the CVC. The electret properties of PS largely depend on the terms of its formation, surface condition and the external atmosphere [17].

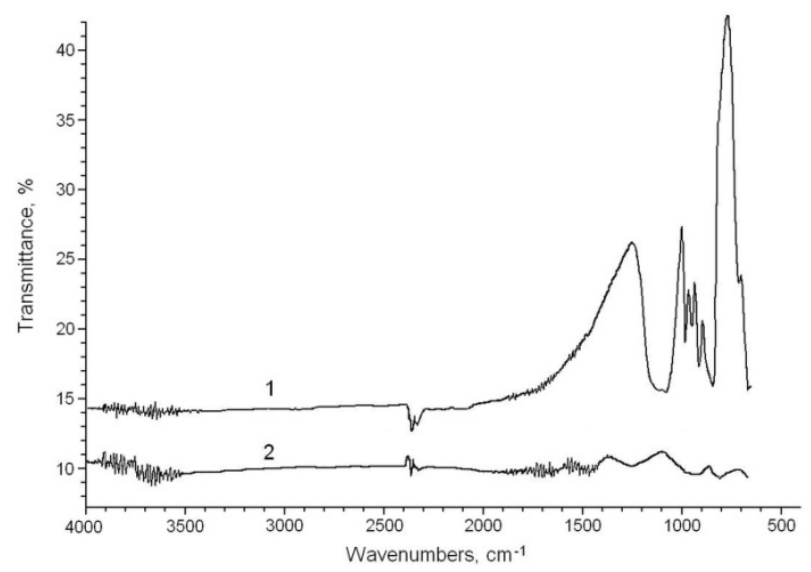

Figure 6. FT-IR spectrum of reference PS - Si (1) and ammonia modified (2) PS - Si structures

\section{Conclusion}

Thus it has been shown that adsorption of ammonia molecules modifies the electrical properties of 'PS-silicon substrate' structures. Increasing $\mathrm{NH}_{3}$ concentration leads to increase in the conductivity in the case of PS- $n$-Si structure and decreases the same parameter for the PS- $-p$-Si structure. The appearance of photosensitive electric barriers in structures PS- $p$-Si on adsorption of molecules $\mathrm{NH}_{3}$ can be due to inversion of the hole-type conductivity of the nanocrystals PS to the electronic type. The obtained data suggest the possibility of controlling the conductivity type of PS nanocrystals by adsorption of chemically active or polar molecules.

It has been found that adsorption of ammonia molecules leads to the electret properties of the PS. Probable cause of the observed effect is to change the molecular composition of surface nanocrystals of PS on adsorption of ammonia molecules.

The structures based on ammonia modified PS demonstrated the photovoltaic effect in the visible spectral range what extends the prospects of their use as photodetectors. The obtained experimental results can be also used for the development of electronic devices not only based on porous silicon, but also on systems of semiconductor nanocrystals.

\section{REFERENCES}

[1] K. Q. Peng, S. T. Lee. Silicon nanowires for photovoltaic solar energy conversion, Advanced Materials, Vol.23, No.2, 198-215, 2011.

[2] S. Ozdemir, J. Gole. The potential of porous silicon gas sensors, Current Opinion in Solid State and Materials Science, Vol.11, No.5-6, 92-100, 2007.

[3] C. Baratto, G. Faglia, G. Sberveglieri, Z. Gaburro, L. Pancheri, C. Oton, L. Pavesi. Multiparametric porous silicon sensors, Sensors, Vol.2, No.3, 121-126, 2002.

[4] Z. M. Rittersma, W. J. Zaagman, M. Zetstra, W. Benecke. A monitoring instrument with capacitive porous silicon humidity sensors, Smart Materials and Structures, Vol.9, No.3, 351-356, 2000.

[5] M. Chiesa, G. Amato, L. Boarino, E. Garrone, F. Geobaldo, E. Giamello. Reversible insulator-to-metal transition in p+-type mesoporous silicon induced by the adsorption of ammonia, Angewandte Chemie International Edition, Vol.42, No.41, 5032-5035, 2003.

[6] A. S. Vorontsov, L. A. Osminkina, A. E. Tkachenko, E. A. Konstantinova, V. G. Elenskii, V. Yu. Timoshenko, P. K. Kashkarov. Modification of the properties of porous silicon on adsorption of iodine molecules, Semiconductors, Vol.41, No.8, 953-957, 2007.

[7] I. B. Olenych. Electrical and photoelectrical properties of iodine modified porous silicon on silicon substrates, Journal Semiconductor Physics, Quantum Electronics \& 
Optoelectronics, Vol.15, No.4, 382-385, 2012.

[8] I. B. Olenych, L. S. Monastyrskii, O. I. Aksimentyeva, B. S. Sokolovskii. Effect of bromine adsorption on the charge transport in porous silicon - silicon structures, Electronic Materials Letter, Vol.9, No.3, 257-260, 2013.

[9] S. C. Erwin, Zu Lijun, M. I. Haftel, A. L. Efros, T. A. Kennedy, D. J. Norris. Doping semiconductor nanocrystals, Nature, Vol.436, No.7047, 91-94, 2005.

[10] D. Mocatta, G. Cohen, J. Schattner, O. Millo, E. Rabani, U. Banin. Heavily Doped Semiconductor Nanocrystal Quantum Dots, Science, Vol.332, No.6025, 77-81, 2011.

[11] A. G. Cullis, L. T. Canham, P. D. J. Calcott. The structural and luminescence properties of porous silicon, Journal of Applied Physics, Vol.82, No.3, 909-965, 1997.

[12] Y. Fukuda, K. Furuya, N. Ishikawa, T. Saito. Aging behavior of photoluminescence in porous silicon, Journal of Applied Physics, Vol.82, No.11, 5718-5721, 1997.

[13] A. Borghesi, A. Sassella, B. Pivac, L. Pavesi.
Characterization of porous silicon inhomogeneities by high spatial resolution infrared spectroscopy, Solid State Communications, Vol.87, No.1, 1-4, 1993.

[14] M. Niwano. In-situ IR observation of etching and oxidation processes of Si surfaces, Surface Science, Vol.427-428, 199-207, 1999.

[15] A. V. Pavlikov, L. A. Osminkina, I. A. Belogorokhov, E. A. Konstantinova, A. I. Efimova, V. Yu. Timoshenko, P.K. Kashkarov. Effect of the initial doping level on changes in the free-carrier concentration in porous silicon during ammonia adsorption, Semiconductors, Vol.39, No.11, 1342-1345, 2005.

[16] Yu. A. Vashpanov. Electronic properties of microporous silicon under illumination and with the adsorption of ammonia, Technical Physics Letters, Vol.23, No.6, 448-449, 1997.

[17] L. S. Monastyrskii, T. I. Lesiv, I. B. Olenych. Composition and properties of thin films on porous silicon surface, Thin Solid Films, Vol.343-344, 335-337, 1999. 